\title{
Interaction of the Tsn1 and Tsc2 sensitivity genes with the ToxA and ToxB effector genes in Triticum L. species
}

\author{
N.V. Mironenko ${ }^{1 *}$, O.A. Baranova ${ }^{1}$, N.M. Kovalenko' ${ }^{1}$, O.P. Mitrofanova ${ }^{2}$ \\ ${ }^{1}$ The All-Russian Institute of Plant Protection, St. Petersburg, Russia \\ ${ }^{2}$ The N.I. Vavilov All-Russian Institute of Plant Genetic Resources
}

DOI 10.18699/ICG-PlantGen2019-19

(c) Autors, 2019

* e-mail: nina2601mir@mail.ru

\begin{abstract}
The present study was aimed at determining the presence of the $\operatorname{Tsn} 1$ (5BL) and Tsc2 (2BL) sensitivity genes in Triticum L. species and at assessing the response of wheat accessions to infection with Pyrenophora tritici-repentis isolates with the complementary effector genes ToxA and ToxB. It was shown that the diploid species T. urartu, T. boeoticum and $T$. monococcum ( $2 n=2 x=14$, A genome) had no Tsn1 and Tsc2 genes and showed no susceptibility reaction. The gene-for-gene interaction of Tsc2-ToxB was observed for the tetraploid species T. aethiopicum and T. turgidum $(2 n=4 x=28, A B)$. Despite the presence of the $T s c 2$ gene, the accessions of T. dicoccoides and T. dicoccum were resistant to the ToxB⿱ $B^{+}$ isolate. Different types of Tsc2-ToxB and Tsn 1-ToxA interactions were detected for accessions of other tetraploid and hexaploid wheat species $(2 n=6 x=42, A B D)$. The paper discusses possible causes of discrepancies between the observed and expected plant phenotypic reactions, which were determined by the interaction of the $T s n 1$ and $T s c 2$ sensitivity genes with the isolates containing the ToxA and ToxB effector genes.

Key words: wheat species; tan spot; effectors; Tsn1 and Tsc2 sensitivity genes; gene-forgene interactions.
\end{abstract}

\section{Introduction}

Tan spot caused by Pyrenophora tritici-repentis is a harmful disease spread worldwide. The three known genes of sensitivity to PtrToxA, PtrToxB, and PtrToxC toxins in P. triticirepentis are $T_{s n} 1, T s c 2$ and $T s c 1$, respectively. The Tsn1-Ptr ToxA, Tsc2-Ptr ToxB, and Tsc1-Ptr ToxC interactions have all been shown to play a significant role in the development of tan spot in common wheat (Triticum aestivum L.) (Faris et al., 2013; Kariyawasam et al., 2016).

The wheat sensitivity genes $T s n 1$ and $T s c 2$, as well as the Tox $A$ and ToxB effector genes encoding PtrToxA and PtrToxB toxins, have been cloned and used for developing gene-specific primers (Faris et al., 2010; Abeysekara et al., 2010; Andrie et al., 2007).

The purpose of the present study was to identify the presence of the $T s n 1$ (5BL) and $T s c 2$ (2BL) sensitivity genes in Triticum L. species and to reveal their response to infection with isolates possessing the Tox $A$ and ToxB complementary effector genes.

\section{Materials and methods}

All the 72 accessions representing 16 Triticum L. species from the VIR wheat collection were assessed for resistance to two isolates with ToxA $\left(\mathrm{ToxA}^{+}\right)$originating from Kazakhstan and Russia, and one ToxB ${ }^{+}$from Greece. Pathogen specific primers were used for PCR detection of $P$. tritici-repentis isolates (Antoni et al., 2010). ToxA and ToxB identification in fungal isolates was performed using gene-specific primers (Andrie et al., 2007). The Tsn 1 sensitivity gene was identified using functional allele-specific primers (Faris et al., 2010), and $T s c 2$, by using the XBE444541 marker (Abeysekara et al., 2010).

The procedure of resistance determination was based on determining the size of necrotic and chlorotic spots that formed after inoculation of seedling leaves with a conidial suspension. The detached leaf assays in benzimidazole $(40 \mathrm{mg} / \mathrm{l})$ were used. In the present work, the reaction of wheat accessions to the inoculation with $\mathrm{ToxA}^{+}$isolates (necrosis) and to the ToxB $^{+}$isolate (chlorosis) below 2 points were considered as resistance.

\section{Results and discussion}

According to the gene-for-gene model, necrosis and/or chlorosis are observed on wheat leaves when both the plant and pathogen have the dominant genes Tsn1/ToxA and/or Tsc2/ ToxB , respectively. Neither Tsn1 nor Tsc2 genes nor sensitivity reactions have been detected in all accessions of the diploid species T. urartu, T. boeoticum and T. monococcum. The gene-for-gene Tsc2-ToxB interaction was observed in the tetraploid wheats T. aethiopicum and T. turgidum, as well as in most accessions of the hexaploid wheats T. sphaerococcum and $T$. compactum, which had $T s c 2$ and were sensitive to the ToxB $^{+}$isolate.

Out of $14 T$. dicoccoides and $T$. dicoccum accessions, 12 were resistant to the $\mathrm{ToxB}^{+}$isolate despite the presence of the $T s c 2$ gene. Such a 'gene/plant reaction' link can be explained either by a mutation in the gene that disrupted its expression or by the presence in accessions of other gene(s) homologous to Tsc2.

When evaluating the Tsn1-Tox $A$ interaction, 8 accessions of different tetra- and hexaploid wheats (T. aethiopicum, T. durum, T. sphaerococcum) aroused particular interest. The dominant allele $T_{s n} 1$ was not detected in these accessions, but strong necrosis was observed after infection with the ToxA $^{+}$isolate, which is possibly due to the existence of other unknown susceptibility and effector genes. A number of accessions, in constrast, displayed the presence of $T_{s n} 1$ and the absence of susceptibility reactions. The reasons may be the same as in the case with Tsc2/ToxB interaction in T. dicoccoides and $T$. dicoccum. The studied accessions of T. macha and T. spelta were resistant to the Tox $\mathrm{A}^{+}$and ToxB ${ }^{+}$isolates, 
while no $T s n 1$ or $T s c 2$ genes were identified in them, with one exception.

Deviations from the expected gene-for-gene relationships in common wheat, which were observed among the cultivars of T. aestivum (Mironenko, Kovalenko, 2018), can have an explanation that the Tsn1-ToxA interaction may be epistatic to the production of other necrotrophic effectors, depending on the genetic background of the sensitive host (Manning, Ciuffetti, 2015). The authors believe that perhaps some wheat genotypes possess factors that lead to altered expression levels of the ToxA gene through epistasis, or in some way inhibit the recognition of ToxA by $T s n 1$ in plants inoculated with fungal spores.

The Tsc2-ToxB interaction is known to play a significant role in conferring susceptibility in tetraploid (Virdi et al., 2016) and hexaploid wheats (Abeysekara et al., 2010), while the Tsn1ToxA interaction is not a significant factor for the development of tan spot in durum cultivars (Virdi et al., 2016). According to some researchers, the Tsn1-ToxA interaction can play a major or a minor role, or have no effect at all for common wheat, depending on the genetic background (Faris et al., 2013).

The analyzed accessions of T. timopheevii and T. araraticum ( $2 n=4 x=28$, GA genome) have $T s c 2$, but not $T s n 1$; they were found to be resistant to the Tox $\mathrm{A}^{+}$and $\mathrm{ToxB}^{+}$isolates, which suggests the presence of a gene (genes) homologous to $T s c 2$ in these accessions.

\section{Conclusions}

It is believed that the ToxA gene appeared in P. tritici-repentis as a result of horizontal transfer from another wheat pathogen, Stagonospora nodorum (Friesen et al., 2006). Therefore, Tsn 1 is the major determinant for sensitivity to both $S$. nodorum blotch (SNB) and tan spot. The ToxA gene in P. tritici-repentis is less expressed than the identical gene SnToxA in $S$. nodorum during wheat infection (Virdi et al., 2016). The high role of the Tsn1-ToxA interaction was shown for the SNB manifestation in both hexaploid and tetraploid wheat (Liu et al., 2006).

According to our data, the Tsn1-ToxA interaction does not always play a significant role in conferring susceptibility to tan spot in hexaploid wheats in the wheat- $P$. tritici-repentis pathosystem, and has no effect on this disease manifestation in durum wheat, in contrast to the Tsn1-ToxA interaction in the wheat-S. nodorum pathosystem. We believe that one of the reasons for these differences may be the low expression of the ToxA gene in P. tritici-repentis, which was introduced into this pathogen as part of the alien translocation from another wheat pathogen, S. nodorum (Friesen et al., 2006).

\section{References}

Abeysekara N.S., Friesen T.L., Liu Z., McClean P.E., Faris J.D. Marker development and saturation mapping of the Tan Spot PtrToxB sensitivity locus Tsc2 in hexaploid wheat.Plant Genome. 2010;3(3): 179-189. DOI 10.3835/plantgenome2010.07.0017.

Andrie R.M., Pandelova I., Ciuffetti L.M. A combination of phenotypic and genotypic characterization strengthens Pyrenophora triticirepentis race identification. Phytopathol. 2007;97:694-670. DOI 10.1094/PHYTO-97-6-0694.

Antoni E.A., Rybak K., Tucker M.P. et al. Ubiquity of ToxA and absence of ToxB in Australian populations of Pyrenophora tritici-repentis. Austral. Plant. Pathol. 2010;39:63-68. www.publish.csiro. au/journals/app.

Faris J.D., Liu Zh., Xu S.S. Genetics of tan spot resistance in wheat. Theor. Appl. Genet. 2013;126:2197-2217. DOI 10.1007/s00122013-2157-y.

Faris J.D., Zhang Z., Lu H.J., Lu S.W., Reddy L. et al. A unique wheat disease resistance-like gene governs effector-triggered susceptibility to necrotrophic pathogens. Proc. Natl. Acad. Sci. USA. 2010;107: 13544-13549. Doi.org/10.1073/pnas.1004090107.

Friesen T.L., Stukenbrock E.H., Liu Z., Meinhardt S., Ling H. et al. Emergence of a new disease as a result of interspecific virulence gene transfer. Nature Genetics. 2006;38(8):953-956. Doi.org/10.1038/ ng1839.

Kariyawasam G.K., Carter A.H., Rasmussen J.B., Faris J., Xu S.S., Mergoum M., Liu Z. Genetic relationships between race-nonspecific and race-specific interactions in the wheat - Pyrenophora triticirepentis pathosystem. Theor Appl Gen. 2016;129:897-908. DOI 10.1007/s00122-016-2670-x.

Lamari L., Strelkov S.E., Yahyaoui A., Orabi J., Smith R.B. The identification of two new races of Pyrenophora tritici-repentis from the host centre of diversity confirms a one-to-one relationship in tan spot of wheat. Phytopathol. 2003;93:391-396.

Liu Z., Friesen T.L., Ling H., Meinhardt S.W., Oliver R.P., Rasmussen J.B., Faris J.D. The Tsn1-ToxA interaction in the wheat-Stagonospora nodorum pathosystem parallels that of the wheat-tan spot system. Genome. 2006;49(10):1265-73. DOI 10.1139/g06-088.

Manning V.A., Ciuffetti L.M. Necrotrophic effector epistasis in the $P y$ renophora tritici-repentis-wheat interaction. PLOS ONE. 2015;10(4): e0123548. DOI 10.1371/journal.pone.0123548.

Mironenko N.V., Kovalenko N.M. Peculiarities of interaction of Tsn 1 and ToxA genes in Triticuma estivum - Pyrenophora tritici-repentis pathosystem. PlantProtectionNews. 2018;2(96):12-16. (in Russian)

Virdi S.K., Liu Z., Overlander M.E. New insights into the roles of host gene-necrotrophic effector interactions in governing susceptibility of durum wheat to tan spot and Septoria nodorum blotch. G3. 2016; 6:4139-4150. DOI 10.1534/g3.116.036525/-/DC1.

Acknowledgements. The work is supported by the RFBR grant 1804-00128a and Comprehensive Program of Scientific Research “Development of selection and processing of grain crops"

Conflict of interest. The authors declare no conflict of interest. 